\title{
Influence of the C-terminal domain on the bioluminescence activity and color determination in green and red emitting beetle luciferases and luciferase-like enzyme
}

\author{
V. R. Bevilaqua ${ }^{1} \cdot$ Mariele C. Carvalho $^{1} \cdot$ Gabriel F. Pelentir $^{2} \cdot$ Atílio Tomazini $^{3} \cdot$ Mario Murakami $^{3} \cdot$ Vadim R. Viviani $^{1,2,4}$
}

Received: 4 November 2020 / Accepted: 15 December 2020 / Published online: 19 January 2021

(c) The Author(s), under exclusive licence to European Photochemistry Association,European Society for Photobiology 2021

\begin{abstract}
Beetle luciferases catalyze the bioluminescent oxidation of D-luciferin, producing bioluminescence colors ranging from green to red, using two catalytic steps: adenylation of D-luciferin to produce D-luciferyl-adenylate and PPi, and oxidation of D-luciferyl-adenylate, yielding AMP, $\mathrm{CO}_{2}$, and excited oxyluciferin, the emitter. Luciferases and CoA-ligases display a similar fold, with a large $\mathrm{N}$-terminal domain, and a small $\mathrm{C}$-terminal domain which undergoes rotation, closing the active site and promoting both adenylation and oxidative reactions. The effect of $\mathrm{C}$-terminal domain deletion was already investigated for Photinus pyralis firefly luciferase, resulting in a red-emitting mutant with severely impacted luminescence activity. However, the contribution of C-terminal in the bioluminescence activities and colors of other beetle luciferases and related ancestral luciferases were not investigated yet. Here we compared the effects of the C-terminal domain deletion on green-emitting luciferases of Pyrearinus termitilluminans (Pte) click beetle and Phrixothrix vivianii railroadworm, and on the red-emitting luciferase of Phrixothrix hirtus railroadworm and luciferase-like enzyme of Zophobas morio. In all cases, the domain deletion severely impacted the overall bioluminescence activities and, slightly less, the oxidative activities, and usually red-shifted the bioluminescence colors. The results support the involvement of the C-terminal in shielding the active site from the solvent during the light emitting step. However, in Pte luciferase, the deletion caused only a $10 \mathrm{~nm}$ red-shift, indicating a distinctive active site which remains more shielded, independently of the $\mathrm{C}^{\prime}$-terminal. Altogether, the results confirm the main contribution of the C-terminal for the catalysis of the adenylation reaction and for active site shielding during the light emitting step.
\end{abstract}

Keywords Luciferases $\cdot$ CoA-ligases $\cdot$ C-terminal $\cdot$ Bioluminescence

Vadim R. Viviani

viviani@ufscar.br

1 Graduate Program of Evolutive Genetics and Molecular Biology, Federal University of São Carlos (UFSCar), São Carlos, Brazil

2 Department of Physics, Chemistry and Mathematics, Federal University of São Carlos (UFSCar), Sorocaba, Brazil

3 Brazilian Biorenewables National Laboratory (LNBR), Brazilian Center for Research in Energy and Materials (CNPEM), Campinas, SP, Brazil

4 Graduate Program of Biotechnology and Environmental Monitoring, Federal University of São Carlos (UFSCar), Rod. João Leme Dos Santos, SP 264, Km 110, Itinga, Sorocaba, SP, Brazil

\section{Introduction}

Beetle luciferases catalyze the bioluminescent oxidation of D-luciferin producing distinct bioluminescence colors, ranging from green to red [1]. The bioluminescence reaction involves two catalytic steps: the adenylation of D-luciferin producing D-luciferin-adenylate $\left(\mathrm{LH}_{2} \mathrm{AMP}\right)$, pyrophosphate and (2) the oxidation of $\mathrm{LH}_{2} \mathrm{AMP}$, yielding a dioxetanone intermediate which spontaneously cleaves yielding carbon dioxide, AMP and excited oxyluciferin, the emitter [1,2]. The first half-reaction is universally catalyzed by the CoAligases superfamily of enzymes [3,4], which catalyze the same adenylation of distinct carboxylic substrates, producing the adenylate intermediate [5]. However, in beetle luciferases the second half-reaction, which in most CoA-ligases consists of the thioesterification of carboxylic substrate with 
CoA, is replaced by the oxidative reaction which yields light emission [1].

The three-dimensional structure of firefly luciferases in the absence and presence of substrates and products and of some CoA-ligases was solved by X-ray crystallography $[6,7]$. This superfamily of enzymes displays similar threedimensional structure, with a large $\mathrm{N}$-terminal and a small $\mathrm{C}$-terminal domains. The $\mathrm{N}$-terminal domain includes the ATP and carboxylic substrate binding sites, whereas the surface of the $\mathrm{N}$-terminal domain displays a surface channel which is confluent with the ATP-binding site, that upon the C-terminal rotation is closed, accommodating the CoA substrate [8]. The luciferin binding site in firefly luciferases has been identified, and several studies have identified important residues involved in catalysis and bioluminescence color determination [9]. During the catalytic steps, the C-terminal domain undergoes rotation, closing the active site and promoting the adenylation and thioesterification reactions $[3,10]$. The C-terminal rotation was also shown to be important for the bioluminescent oxidative reaction in firefly luciferase [11].

The effect of C-terminal domain deletion on luciferase function was previously investigated in the $\mathrm{pH}$-sensitive $\mathrm{Pho}$ tinus pyralis firefly luciferase [12]. The deletion mutant loses most of its bioluminescence activity, displaying much lower affinity for luciferin and ATP with a much slower luminescence reaction kinetics, and emission spectrum in the red region. However, it not clear how much the C-terminal influences the bioluminescence color and activity in other beetle luciferases. Furthermore, it would be interesting to compare the importance of C-terminal for the bioluminescence and oxidative activities in luciferase-like enzyme of Zophobas morio mealworm. This enzyme is not a true luciferase, but a generalistic CoA-ligase of unknown biological function, which in presence of ATP and exogenous D-luciferin emits weak chemiluminescence in the red region. Because of its alternative luminescence reaction, this enzyme has been considered a reasonable model of protoluciferase and missing link from dark CoA-ligases to bright luciferases.

Thus, to better understand the influence of the C-terminal in the catalysis of bioluminescence reactions and bioluminescence spectra of beetle luciferases from different families emitting distinct bioluminescence colors and related enzymes, we compared the effect of $\mathrm{C}$-terminal domain deletion in the green-emitting luciferases from Pyrearinus termitilluminans larval click beetle (Elateridae) and Phrixothrix vivianii (Phengodidae), in the red-emitting luciferase from $P$. hirtus railroadworm (Phengodidae), and in the luciferase-like enzyme from Zophobas morio (Tenebrionidae).

\section{Results and discussion}

\subsection{Luciferase expression}

All luciferases were expressed in E.coli BL21 cells, extracted and some of them purified (Fig. 1). As expected, the $\mathrm{C}^{\prime}$-terminal deletion-mutant luciferases had their MW reduced $\sim 10 \mathrm{kDa}$ in relation to the wild-type enzymes. Upon deletion of the C-terminal domain, the luciferase-like enzyme from $Z$. morio molecular weight was reduced from 59 to $47.7 \mathrm{kDa}$.

Although the red-emitting luciferase of P.hirtus railroadworm (PxRE) showed higher expression levels than the $P$. termitilluminans larval click beetle luciferase and its deletion mutant, most of the protein was recovered in the pellet, indicating that this mutant enzyme was more insoluble than the other luciferases. Apparently, the C-terminal is important for the solubility of this enzyme. Because most of these luciferase deletion mutants were very unstable and quickly lost the activity during the purification steps, we measured the luminescence activities only in crude extracts.

\subsection{Effect of C-terminal deletion on overall bioluminescence activity}

We measured the overall bioluminescence activity of the C-terminal deletion mutants of 3 beetle luciferases emitting distinct bioluminescence (BL) colors and a luciferaselike enzyme. The overall bioluminescence activity reflects the product of the first adenylation reaction and the second oxidative one. In all cases, the bioluminescence activity starting with D-luciferin and ATP as substrates was severely impaired (Fig. 2 and Table 1). Among the deletion mutants, those of P.hirtus railroadworm red emitting and

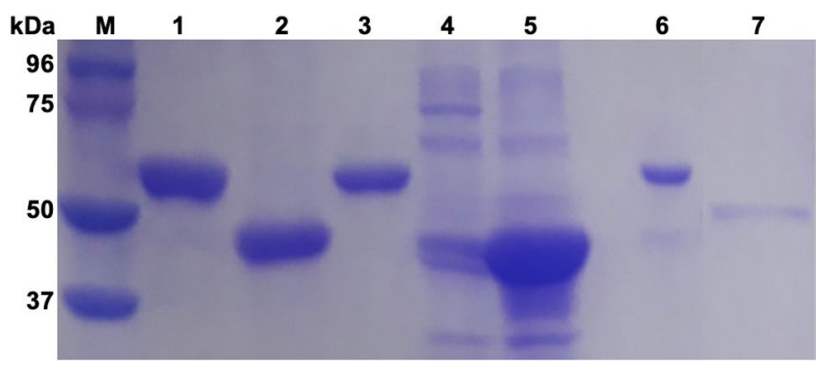

Fig. 1 SDS-PAGE of purified wild-type luciferase and their C-terminal domain deletion mutants: (M) Molecular weight markers; (1) wild-type $Z$. morio luciferase-like enzyme, (2) $\mathrm{C}^{\prime}$-terminal deletion mutant of $Z$. morio luciferase-like enzyme; (3) wild-type $P$. hirtus railroadworm red-emitting luciferase; (4) $\mathrm{C}^{\prime}$-terminal deletion mutant of $P$. hirtus red-emitting luciferase; $(\mathbf{5})$ insoluble fraction of $\mathrm{C}^{\prime}$-terminal deletion mutant of $P$. hirtus red-emitting luciferase; (6) wild-type $P$. termitilluminans larval click beetle luciferase, and (7) $\mathrm{C}^{\prime}$-terminal deletion mutant $P$. termitilluminans luciferase 
Fig. 2 Overall relative bioluminescence and oxidative activities of C-terminal domain deletion mutants and WT luciferases and luciferase-like enzyme: (dark grey) with D-luciferin and ATP; (light grey) with luciferyladenylate. (PxRE) Phrixothrix hirtus railroadworm red-emitting luciferase; (Pte) Pyrearinus termitilluminans larval click beetle luciferase; (Zop) Zophobas morio mealworm luciferaselike enzyme

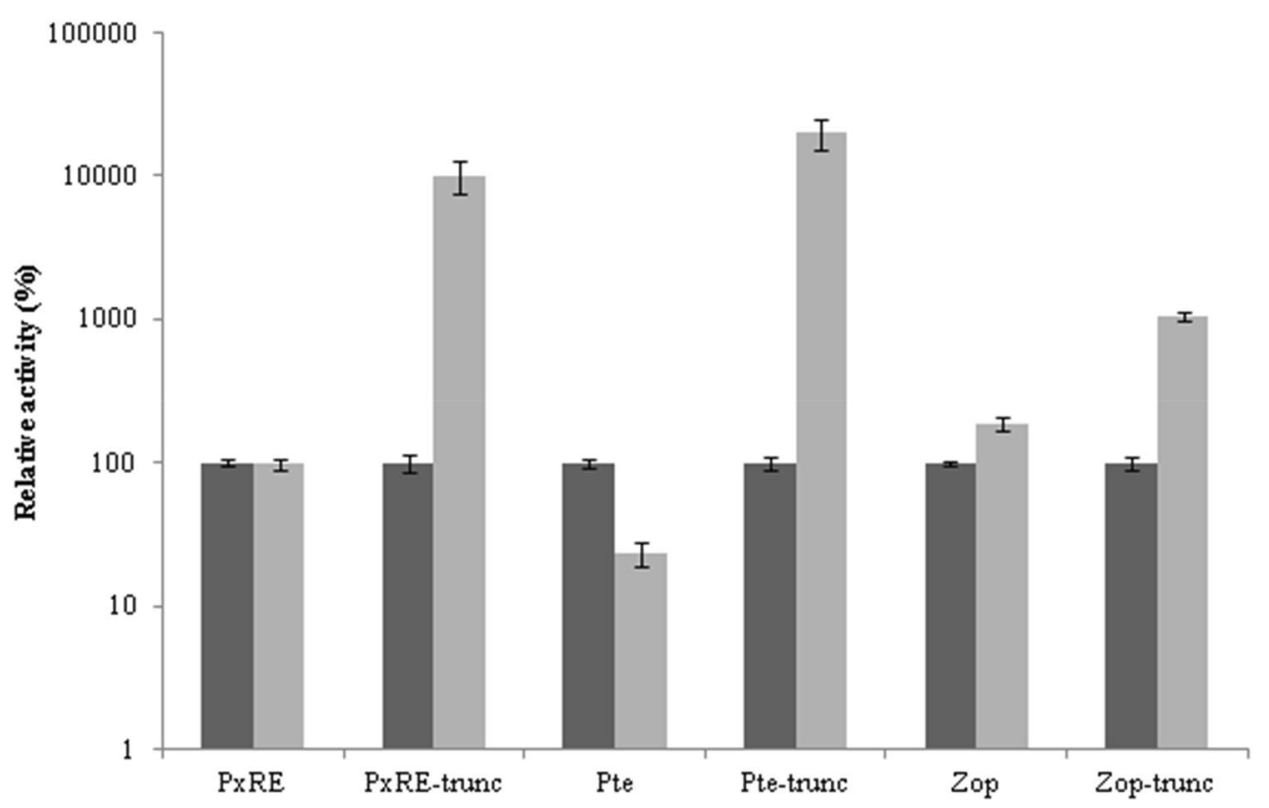

Table 1 Bioluminescence and kinetics properties of luciferase C-terminal deletion mutants

\begin{tabular}{|c|c|c|c|c|c|c|}
\hline Luciferase & $\begin{array}{l}\mathrm{A}_{(\mathrm{LH} 2+\mathrm{ATP})}\left(10^{6} \mathrm{cps} /\right. \\
\mathrm{mg}) \pm \mathrm{SD}^{*}\end{array}$ & $\begin{array}{l}\mathrm{A}_{(\mathrm{LH} 2 \mathrm{AMP})}\left(10^{6} \mathrm{cps} /\right. \\
\mathrm{mg}) \pm \mathrm{SD}^{*}\end{array}$ & $\mathrm{t}_{1 / 2 \text { (LH2+ATP) }}(\mathrm{s})$ & $\mathrm{t}_{1 / 2 \text { (LH2AMP) }}(\mathrm{s})$ & $\begin{array}{l}\lambda_{\max }(\mathrm{nm}) \\
(\mathrm{LH} 2+\mathrm{ATP})\end{array}$ & $\begin{array}{l}\lambda_{\max } \\
(\mathrm{nm}) \\
(\mathrm{LH} 2 \mathrm{AMP})\end{array}$ \\
\hline \multicolumn{7}{|l|}{ ELATERIDAE } \\
\hline \multicolumn{7}{|l|}{ P.termitilluminas } \\
\hline WT & $123,614 \pm 9023$ & $23,363 \pm 1027$ & 290 & 132 & 546 & 590 \\
\hline $\mathrm{C}^{\prime}$ domain deletion & $0.94 \pm 0.09$ & $188 \pm 34$ & 1080 & 144 & 562 & 594 \\
\hline \multicolumn{7}{|l|}{ PHENGODIDAE } \\
\hline \multicolumn{7}{|l|}{ Phrixothrix vivianii } \\
\hline WT & & & 2310 & & 548 & 548 \\
\hline $\mathrm{C}^{\prime}$ domain deletion & $0.118 \pm 0.048$ & $0,355 \pm 0.025$ & - & 25.5 & - & 600 \\
\hline \multicolumn{7}{|l|}{ Phrixothrix hirtus } \\
\hline WT & $29,838 \pm 1641$ & $27,689 \pm 2685$ & 10 & 50 & 626 & 626 \\
\hline $\mathrm{C}^{\prime}$ domain deletion & $0.32 \pm 0.047$ & $102 \pm 23$ & - & 70 & - & 627 \\
\hline \multicolumn{7}{|l|}{ TENEBRIONIDAE } \\
\hline \multicolumn{7}{|c|}{$\begin{array}{l}\text { Zophobas morio } \\
\text { Luciferase-like enzyme }\end{array}$} \\
\hline WT & $800 \pm 32$ & $1,500 \pm 95$ & 138 & - & 602 & 602 \\
\hline $\mathrm{C}^{\prime}$ domain deletion & $4.8 \pm 0.87$ & $50 \pm 6$ & & - & 623 & 623 \\
\hline
\end{tabular}

* SD is the standard deviation

$P$. vivianii railroadworm green-emitting luciferases did not displayed measurable luminescence activity, whereas that of $P$. termitilluminans larval click beetle displayed weak measurable activity. Noteworthy, the luciferase-like enzyme of Zophobas morio mealworm, which naturally displays very weak red bioluminescence activity with the non-natural substrate D-luciferin, still displayed a weak, but measurable activity $(\sim 2 \%$ of the wild-type enzyme activity).

\subsection{Effect of the C-terminal deletion on the oxidative activity}

The bioluminescence (BL) activity with luciferyl-adenylate, which reflects only the oxidative reaction, was also severely impacted in all the luciferases deletion mutants (Fig. 2). However, despite being impacted, the oxidative activities were comparatively higher in relation to the overall activities, indicating that the $\mathrm{C}$-terminal deletion impacted more 
severely the adenylation activity rather than the oxidative activity. A similar result was also previously reported for $P$. pyralis luciferase deletion mutant [13].

A slightly higher bioluminescence activity with luciferyladenylate in relation to the overall bioluminescence activity was also observed for the luciferase-like enzyme deletion mutant (Fig. 2).

\subsection{Luminescence kinetics}

The luminescence kinetics of beetle luciferases starting the reaction with D-luciferin and ATP, or with luciferyladenylate, as substrates, are remarkably different, usually becoming much faster with the latter substrate.

The kinetics of luminescence reaction also considerably changed upon $\mathrm{C}^{\prime}$-terminal domain deletion in relation to the respective wild-type luciferases, as shown by Fig. 3 and by the half-life of the luminescence decay $\left(\mathrm{t}_{1 / 2}\right)$ shown in Table 1. For P.termitilluminans click beetle luciferase, the deletion of the C-terminal domain resulted in a slightly slower luminescence kinetic profile in relation to that of the wild-type enzyme. However, as with luciferyl-adenylate, the decay became much faster, similar to that observed for the wild-type enzyme with the same substrate.
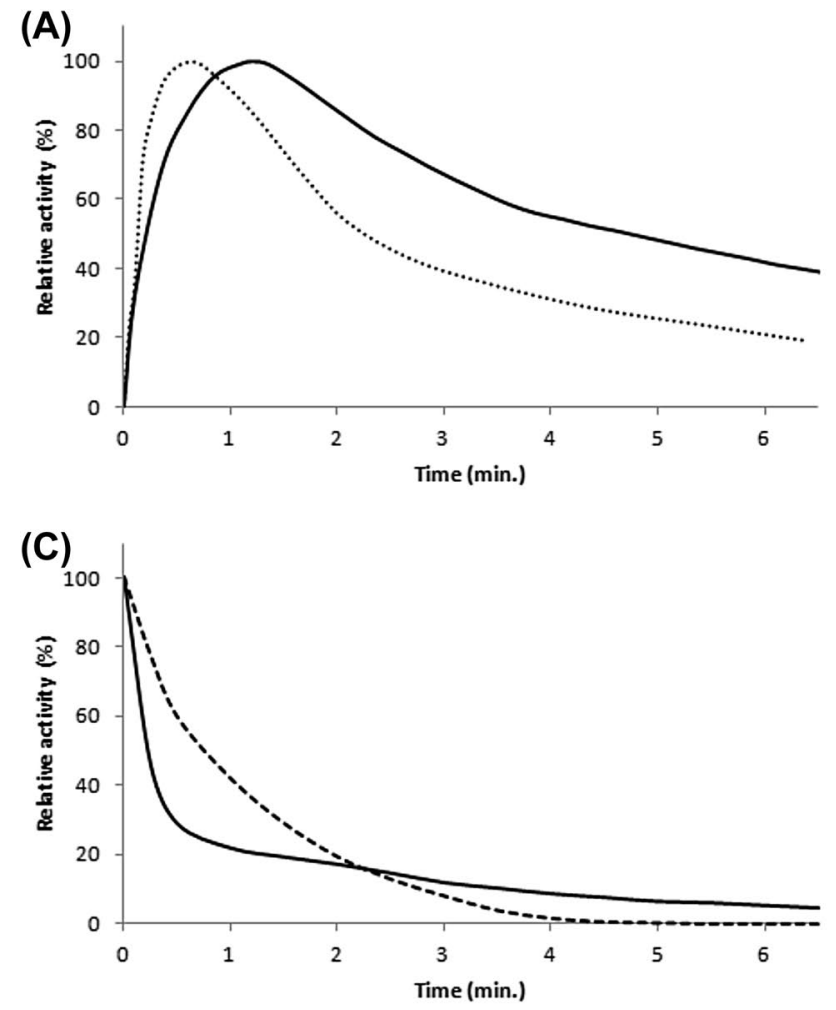

Fig. 3 Kinetics of luminescence reaction of beetle luciferase C'-deletion mutants: (a) P.termitilluminans WT; (b) P.termitilluminans deletion mutant; (c) Phrixothrix hirtus red-
The luminescence kinetics of Phrixotrix hirtus railroadworm red-emitting luciferase is naturally flash-like using either D-luciferin and ATP, or luciferyl-adenylate. Noteworthy, the kinetic profile of the deletion mutant of this luciferase with luciferyl-adenylate became slightly slower when compared to the kinetics of the wild-type luciferase starting with either D-luciferin and ATP, or with luciferyladenylate. For Phrixothrix vivianii green emitting luciferase, the luminescence kinetics was previously shown to be very slow with D-luciferin and ATP and flash-like with luciferyl-adenylate [14]. Its C-terminal deletion mutant, however, displays a flash-like kinetics with luciferyladenylate, similarly to those of the respective wild-type luciferase and P.hirtus wild-type luciferase with the same substrate (results not shown).

The slower and more sustained kinetics of the deletion mutants with luciferin and ATP, in relation to the respective wild-type enzymes, indicate that the removal of the C-terminal contributes for promoting the product removal from the active site, avoiding product inhibition responsible for the fast decay.
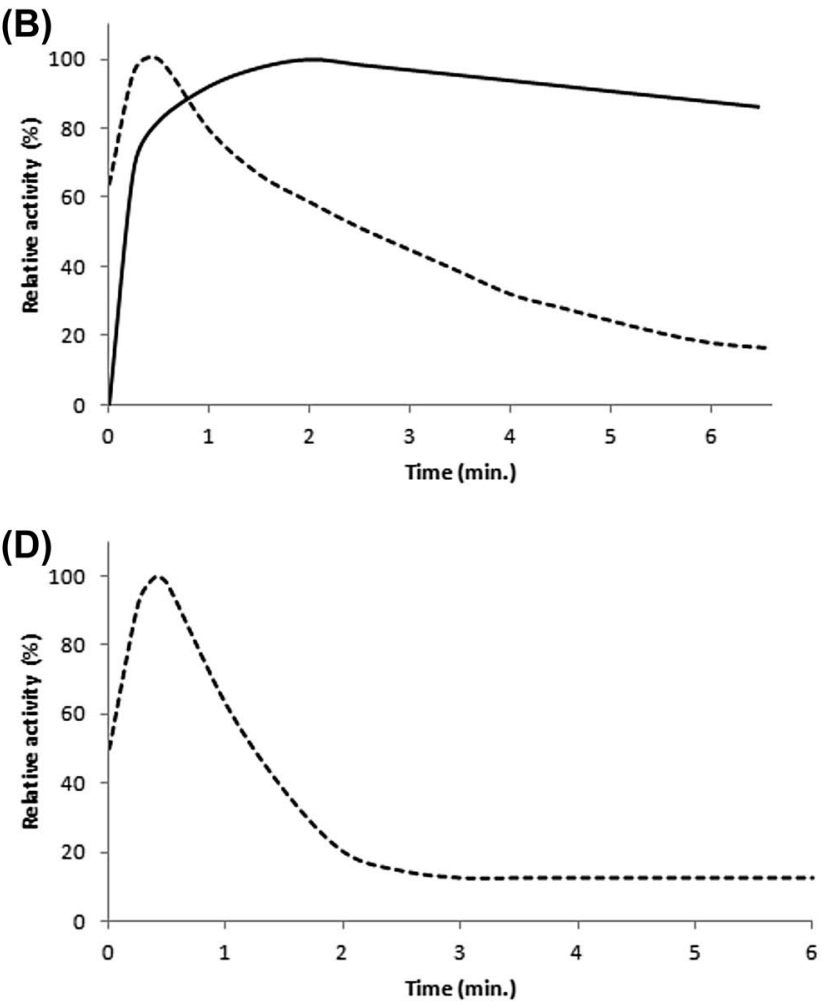

emitting luciferase WT and (d) P. hirtus deletion mutant. (full line) d-luciferin and ATP; (dotted line) Luciferyl-adenylate 


\subsection{Effect on Bioluminescence spectra}

The deletion of the C-terminal domain in Phrixothrix vivianii green-emitting luciferase (PxGR) resulted in orangered emission $(\sim 600 \mathrm{~nm})$. With $P$. hirtus red-emitting luciferase (PxRE), we could not measure the bioluminescence (BL) spectrum with luciferin and ATP because lack of measurable activity, however, we could measure it with luciferyl-adenylate. The BL spectrum of the deletion mutant with luciferyl-adenylate almost overlapped with that of the WT enzyme ( $623 \mathrm{~nm})$ (Fig. 4).

Noteworthy, the BL spectrum of $P$. termitilluminans (Pte) luciferase deletion mutant was only $\sim 16 \mathrm{~nm}$ redshifted in relation to the WT, but still in the green-yellow region, indicating that this this enzyme has a distinct active site, which still remains partially shielded active site from the solvent after the removal of the c-terminal domain.

Furthermore, the BL spectrum with luciferyl-adenylate for both wild-type and its C-terminal deletion mutant were in the orange region $(590 \mathrm{~nm}$; Fig. 4), in contrast with the bioluminescence spectra for the same enzymes using luciferin and ATP as substrates. This is noteworthy because all other beetle luciferases investigated up to date display essentially the same BL spectra either with D-luciferin and ATP or with luciferyl-adenylate, Pte being the only luciferase which displays distinct BL colors using these substrates (luciferin and ATP; $546 \mathrm{~nm}$; luciferyl-adenylate: $590 \mathrm{~nm}$ ). Altogether, the above results indicate that Pte luciferase may display two displaced binding sites for luciferin and its enzymatically formed luciferyl-adenylate, and for exogenously provided luciferyl-adenylate. Alternatively, the enzyme may undergo different conformations when starting the reaction with these substrates. It is possible that the exogenously provided luciferyl-adenylate may not fit well in the same luciferin binding site, explaining the distinct $\mathrm{BL}$ colors.

Finally, the Zophobas morio luciferase-like enzyme deletion mutant displayed considerably red-shifted spectra starting with either ATP or D-luciferin, or with luciferyladenylate (Fig. 4) in relation to the wild-type enzyme, indicating higher exposure of the active site to the solvent upon C-terminal domain deletion.

Taken together, the above bioluminescence spectra and kinetic results with distinct beetle luciferases clearly show that whereas the C-terminal domain is essential for shielding the active site during the adenylation and oxidation catalytic steps, it is not the major determinant of bioluminescence colors. The bottom of the luciferin binding site, as well as the proper fitting of luciferin and excited oxyluciferin inside the luciferin binding site play major roles in bioluminescence spectra modulation.
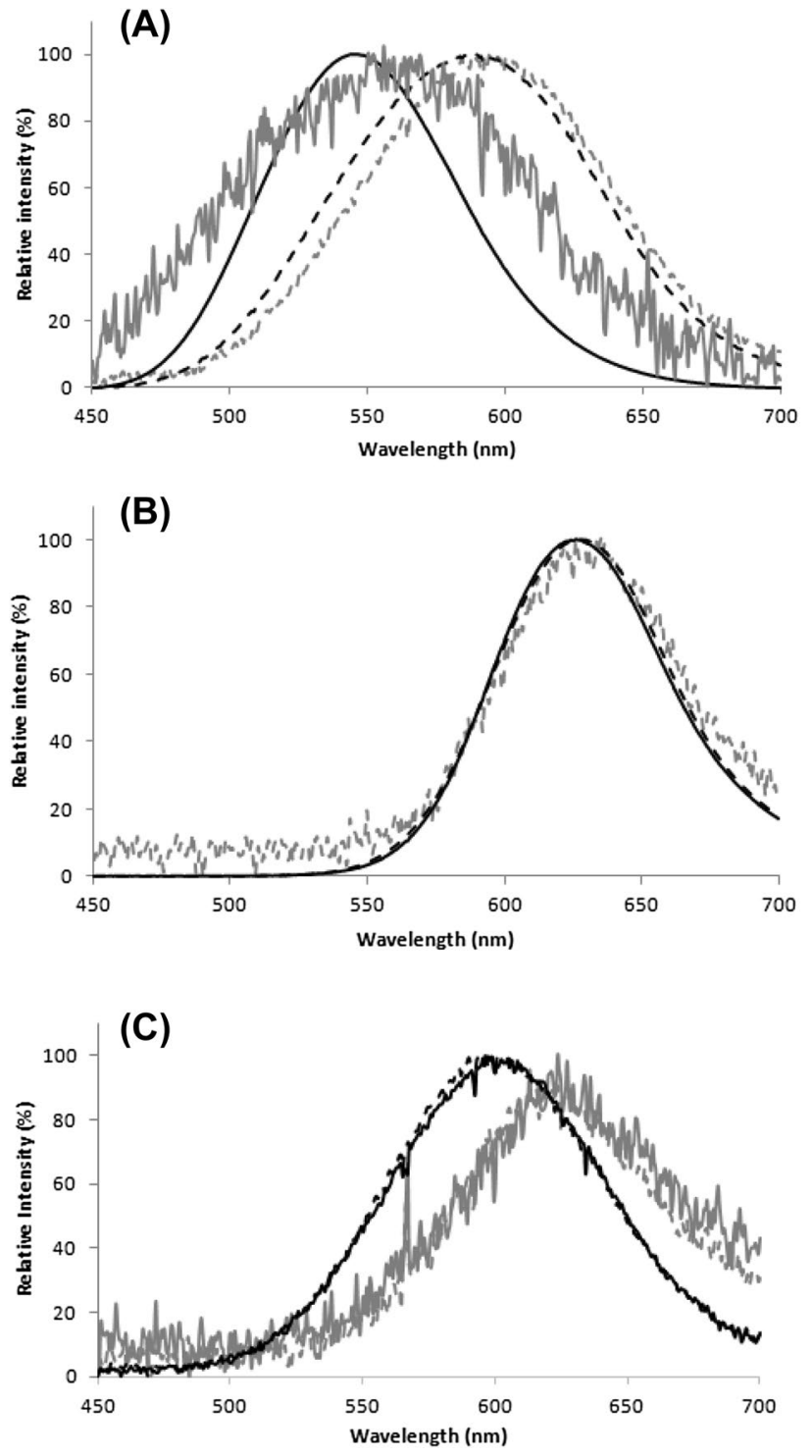

Fig. 4 Bioluminescence spectra of beetle luciferases and their C-terminal domain deletion mutants: (a) Pyrearinus termitilluminans luciferase: (Black line) wild-type with D-luciferin and ATP; (Dashed Black line) wild-type with luciferyl-adenylate; (Grey line) Pte C-deletion mutant with D-luciferin and ATP; (Dashed Grey line) Pte C-deletion mutant with luciferyl-adenylate; (b) Phrixothrix hirtus red-emitting luciferase: (Black line) wild-type luciferase with Luciferin and ATP; (Dashed Black line) wild-type with luciferyl-adenylate; (Dashed Grey line) PxRE C-deletion mutant with luciferyl-adenylate; (c) Zophobas morio luciferase-like enzyme: (Black line) wild-type with D-luciferin and ATP; (Red line) wild-type with luciferyl-adenylate; (Light grey line) wild-type with D-luciferin and ATP and (Dark grey line) C-Deletion mutant with luciferyl-adenylate

\subsection{Modeling of C-terminal domain deletion mutants}

To explain the distinct effects of C-terminal domain deletion in distinct luciferases bioluminescence properties, we made homology based three-dimensional models. The models are 
based on the template structure reported by Nakatsu et al. (2006) [7] for Luciola cruciata firefly luciferase (pdb id: 2D1T) in complex with the high-energy intermediate analog, 5'-O-[N-(dehydroluciferyl)-sulfamoyl]adenosine (DLSA) which simulates luciferyl-adenylate substrate (and product of the first half-reaction with ATP and luciferin).

According to the ab initio models for luciferases and luciferase-like enzyme without the C-terminal domain, it is possible to observe the differences in the $\mathrm{N}$-terminal active site pocket that face the c-terminal domain (Fig. 5). Based on that, Phrixothrix hirtus (PxRE-trunc) and Phrixotrix vivianii (PxGR-trunc) luciferases (Fig. 6a and c) and Zophobas morio luciferase-like enzyme (Zop-trunc) (Fig. 6d) structural models exhibited more exposed pockets when compared to Pyrearinus termitilluminans luciferase deletion mutant (Pte-trunc) (Fig. 5b).

Using an automated blind-docking-server-BINDSURF [15] - the interaction between protein models-ligand was evaluated, where the binding free energy were $-11.2,-9.5$ and $-10.4 \mathrm{kcal} / \mathrm{mol}$ for PxRE-trunc, PxGR-trunc and Zoptrunc with DLSA, respectively. Noteworthy, for Pte-trunc, the automated method was not efficient because the cavity entrance was partially occluded. In this case, the ligand was manually superposed to the site using Pymol.

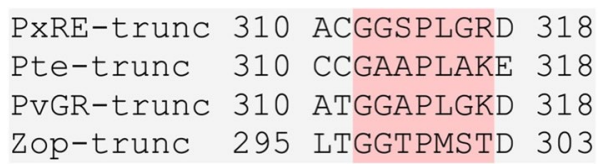

Fig. 6 Multialignement of the loop (red highlight) between residues 312-317 for beetle luciferases and 297-302 for Z. morio luciferaselike enzyme

Two amino acid residues, Thr340 and His242 in the luciferases and the respective Ser325 and Trp227 in luciferase-like enzyme active sites, are located at the entrance to the cavity of the luciferin binding site close to luciferin carboxyl group and phosphate group of ATP [16] (Fig. 6). Another highlight is related to variations in the loop sequence between residues 312 to 317 in the luciferases and 297 to 302 in the luciferase-like enzyme (PxREtrunc: 312-GGSPLG-317; Pte-trunc: 312-GAAPLA-317; PxGR-trunc: 312-GGAPLG-317 and Zop-trunc: 297-GGTPMS-302). The substitutions in such loop could be fundamental to explain the specific kinetic and spectral properties observed in each luciferase (Fig. 6).

The residue Ala313 in Pte-trunc and Gly313 in PxREtrunc, PvGR-trunc and Zop-trunc are located at the entrance

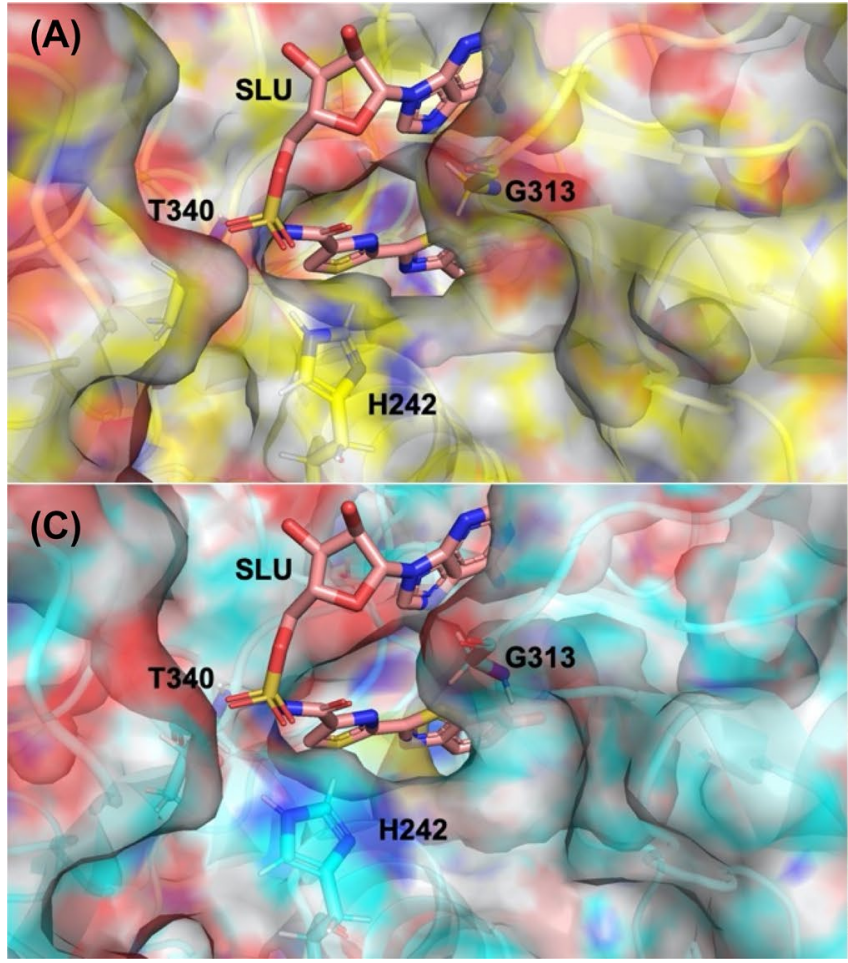

Fig. 5 Docking of the chemical structure of luciferyl-adenylate intermediate analog, DLSA in the crystallographic model of each luciferase and luciferase-like enzyme with C-terminal deletion: (a) Phrixothrix hirtus (PxRE-trunc); (b) Pyrearinus termitilluminans (Pte-trunc); (c) Phrixothrix vivianii (PxGR-trunc); (d) Zophobas

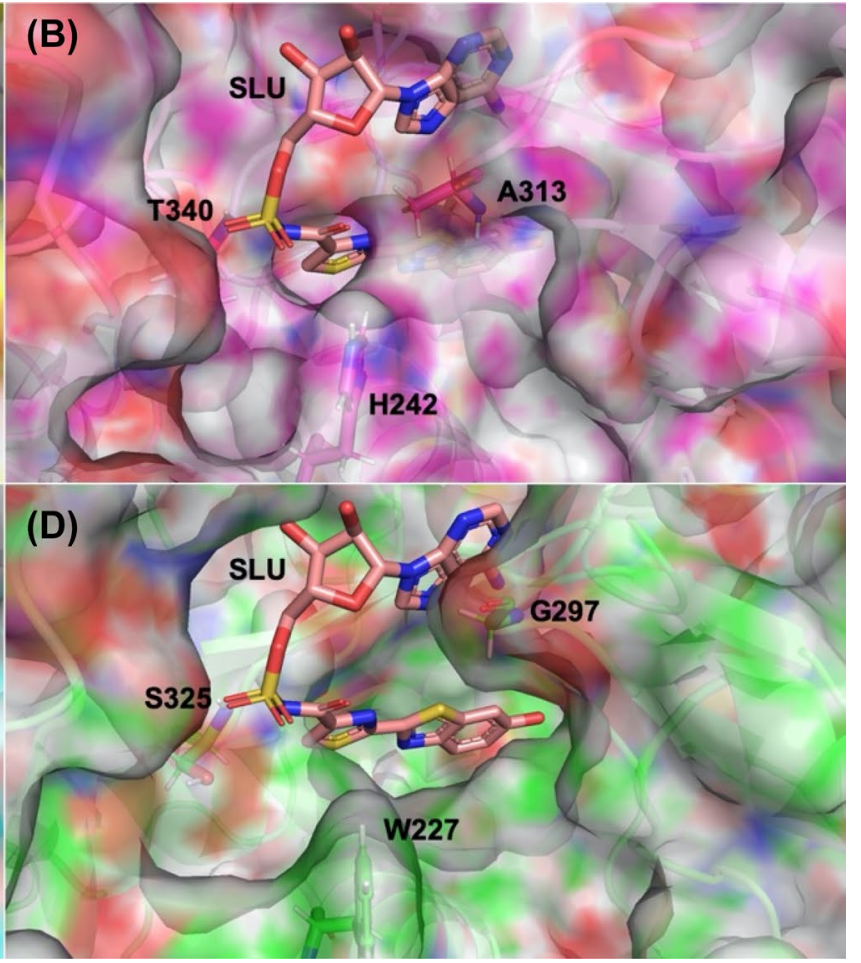

morio (Zop-trunc). For a clearer presentation, only the amino acid residues located at the entrance of the cavity are shown in sticks with secondary structures shown in the cartoon. The surface of the protein is transparent 
to the cavity of the luciferin binding site, close to its carboxyl group and to the phosphate group of ATP. The residue alanine is similar to glycine, but with higher hydrophobicity and side chain volume (because Ala has an additional methyl group). Volume and hydrophobic proprieties could affect the solvent access to the luciferin binding site and affect light emission properties.

The results shown here confirm the distinct active site structure for Pte-trunc indicated by the bioluminescence spectra in the green region and kinetics, suggesting a less exposed active site. In this luciferase, the presence of Ala313 and the dyad of cysteines could indicate greater stability (rigidity) for this loop, partially occluding the luciferin binding site entrance facing the $\mathrm{C}$-terminal domain. The increased rigidity of this flexible segment and the smaller cavity entrance in the active site explains the partial shielding of the active site and the resulting blue-shifted emission even in the absence of C-terminal domain in this enzyme. Furthermore, this more rigid loop in Pte luciferase may also explain why bioluminescence spectra is in the green region when starting the reaction with luciferin and ATP, and orange when starting with exogenously provided luciferyladenylate. The enzymatically formed luciferyl-adenylate may orient the luciferin and the resulting excited oxyluciferin moieties at the bottom of the hydrophobic luciferin binding pocket, resulting in blue-shifted emission. However, the exogenously provided luciferin-adenylate, due to the partially occluded binding site, may have its access limited to such cavity, dislodging luciferin and the resulting excited oxyluciferin to another more exposed and polar site, resulting in red-shifted emission.

Using the Dynamut program we simulated the mutation of this residue. For PxRE-trunc the substitution of A310C showed an improvement in stability of $\Delta \mathrm{G}$ of $0.373 \mathrm{kcal} /$ mol, whereas for Pte-trunc the substitution C310A caused a destabilization of $\Delta \mathrm{G}$ of $-1.139 \mathrm{kcal} / \mathrm{mol}$. For PxGR-trunc the substitution T311C caused a stabilization with $\Delta \mathrm{G}$ of $1450 \mathrm{kcal} / \mathrm{mol}$. For Zop-trunc the respective substitution L295A caused a destabilization of $\Delta \mathrm{G}$ of $-2682 \mathrm{kcal} / \mathrm{mol}$ and for Zop-trunc T296C stabilizes with $\Delta \mathrm{G}$ of $0.779 \mathrm{kcal} /$ mol. Therefore, the cysteine dyad 310-311 is important for the stabilization of Pte-trunc active site, providing an explanation for the differential activity, kinetics and spectra in relation to other luciferases.

These models, support the conformational hypothesis of BL color tuning in beetle luciferases, where a more closed and hydrophobic environment, like in Pte luciferase luciferin binding site, promotes a more blue-shifted emission, whereas a more open and solvent accessible cavity promotes red light emission, as is observed in PxRE and luciferaselike enzyme [16-18]. Moreover, the molecular structures of luciferases, luciferase-like enzyme and the enzyme/ substrate/product interactions depend on dynamic properties such as the rate of reorientation of solvent molecules [16].

\section{Concluding remarks}

The C-terminal domain deletion in beetle luciferases of different families which emit distinct bioluminescence (BL) colors, and in a luciferase-like enzyme, severely impaired overall BL activities and usually red-shifted bioluminescence spectra. The comparatively slightly higher oxidative activities in relation to the overall bioluminescence activities in the deletion mutants, as well as the red-shifted spectra in relation to the wild-type enzymes, support the major involvement of the $\mathrm{C}$-terminal domain in closing and shielding the active site during the adenylation catalytic step and, to a slightly lower extent, for the oxidative and light emitting steps, contributing to provide a closed hydrophobic environment favorable to blue-shifted emission spectra. However, the results with the C-terminal domain deletion of Pte luciferase, which still emits bioluminescence in the green-yellow region, due to more occluded and less accessible luciferin binding site, indicate that the $\mathrm{C}$-terminal domain active site closing effect is not the main factor determining green bioluminescence. Rather, the proper fitting of excited oxyluciferin at the bottom of luciferin binding site plays the most critical role for green light emission.

\section{Experimental}

\subsection{Plasmids and beetle luciferases CDNAs}

The cDNAs for Phrixotrix hirtus red-emitting (PxRE), Pyrearinus termitilluminans green-emitting (Pte) luciferases and Zophobas morio (Zop) luciferase-like enzyme were previously subcloned into pCold-vector (Takara) [19-21]. The cDNA for Phrixotrix vivianii green-emitting luciferase (PxGR) was previously subcloned in pCAN vector [14].

\subsection{Site-directed mutagenesis}

The C'-terminal deletion mutants of Phrixothrix vivianii green-emitting luciferase (PvGR-trunc), Phrixothrix hirtus red-emitting (PxRE-trunc), Pyrearinus termitilluminans green-emitting luciferases (Pte-trunc) and for Zophobas morio luciferase-like enzyme (Zop-trunc) were obtained upon generation of a TAA stop codon near residue 440 of the respective luciferase cDNAs, by site-directed mutagenesis using a Agilent site-mutagenesis kit (Catalog 200518). The plasmids containing the luciferase cDNAs were 
amplified using $P f u$ turbo (Agilent) or Phusion (Thermo) polymerases and two complementary primers containing the desired mutation, using a thermal cycler $(1$ cycle $95{ }^{\circ} \mathrm{C} ; 25$ cycles $95{ }^{\circ} \mathrm{C}, 30 \mathrm{~s} ; 55^{\circ} \mathrm{C}, 1 \mathrm{~min}$ and $68{ }^{\circ} \mathrm{C}$, $7 \mathrm{~min}$ ). After amplification, mutated plasmids containing staggered nicks were generated. The products were treated with $D p n$ I to digest non-mutated parental plasmids, and used directly to transform $E$. coli XL1-Blue cells. The following forward and respective reverse primers were used (the mutation codon is highlighted in bold): (PxRE-CDel: Forward- ATC TAT GTG GTG TAA CGG CTG AAA GAG); (Pte-CDel: Forward-CTA CAT CGT GTA ACG GTA CAA GG; (PxGR-CDel) GCG CCT GCT TAA CTG GAA AAT C; (Zop-CDel): Foward AAGGAAATGTAG TTTAAGTACCAGTCG.

\subsection{Luciferase expression and purification}

For luciferase expression, transformed E. coli BL21-DE3 cells were grown in $100-1000 \mathrm{~mL}$ of $\mathrm{LB}$ medium at $37^{\circ} \mathrm{C}$ up to $\mathrm{OD}_{600}=0.4$, and then induced at $18{ }^{\circ} \mathrm{C}$ with $0.4 \mathrm{mM}$ IPTG during $18 \mathrm{~h}$. For Zophobas morio luciferase-like enzyme, the cells were induced at $16^{\circ} \mathrm{C}$ during $12 \mathrm{~h}$. Cells were harvested by centrifugation at $2500 \mathrm{~g}$ for $15 \mathrm{~min}$ and resuspended in extraction buffer consisting of $0.10 \mathrm{M}$ sodium phosphate buffer, $1 \mathrm{mM}$ EDTA, $1 \mathrm{mM}$ DTT and $1 \%$ Triton $\mathrm{X}-100,10 \%$ glycerol and protease inhibitor cocktail (Roche), $\mathrm{pH} 8.0$, lysed by ultrasonication and centrifuged at $15,000 \mathrm{~g}$ for $15 \mathrm{~min}$ at $4{ }^{\circ} \mathrm{C}$. For the luciferase-like enzyme, the cells were extracted using $50 \mathrm{mM}$ Tris- $\mathrm{HCl} \mathrm{pH} 7.5,10 \%$ glycerol, $1 \%$ Triton-100, $300 \mathrm{mM}$ $\mathrm{NaCl}$ and protease inhibitory cocktail. We also attempted the purification of these c-terminal deletion mutants. The N-terminal histidine-tagged PxRE, PvGR, Pte luciferases and luciferase-like enzyme were further purified by agarose-Nickel affinity chromatography followed by dialysis, according to established procedures $[22,23]$. The concentrations of purified luciferases were between 0.5 and $1 \mathrm{mg} /$ $\mathrm{mL}$, and the estimated purity, according to SDS-PAGE gels were about $90 \%$.

\subsection{Measurement of luciferase activity}

Luciferase bioluminescence activities were measured using an AB2200 (ATTO; Tokyo, Japan) luminometer. The assays of luciferases activities were performed by mixing $5 \mu \mathrm{L}$ of $40 \mathrm{mM}$ ATP/ $/ 80 \mathrm{mM} \mathrm{MgSO}_{4}$ with a solution consisting of 5 $\mu \mathrm{L}$ of crude extract and $85 \mu \mathrm{L}$ of $0.5 \mathrm{mM}$ luciferin in $0.10 \mathrm{M}$ Tris- $\mathrm{HCl} \mathrm{pH} 8.0$ at $22{ }^{\circ} \mathrm{C}$. For the luciferase-like enzyme, $45 \mu \mathrm{L}$ of crude extract were mixed with $45 \mu \mathrm{L}$ of $0.10 \mathrm{M}$ Tris- $\mathrm{HCl}$ buffer $\mathrm{pH}$ 8.0, $5 \mu \mathrm{L}$ of $100 \mathrm{mM}$ d-luciferin and 5 $\mu \mathrm{L}$ of $40 \mathrm{mM}$ ATP and $80 \mathrm{mM} \mathrm{MgSO}_{4}$. All measurements were done in triplicate for at least three independent luciferase preparations, and averages and the standard deviations were reported in the Table 1.

\subsection{Kinetics measurements}

The kinetics of luminescence reaction was performed using a TD-III luminometer (Japan). We used the half-life $\left(\mathrm{T}_{1 / 2}\right)$ in seconds, which is the time from the peak of intensity to half-intensity, as a measure of the decay of luminescence intensity. In the assay for the reaction starting with D-luciferin and ATP, $5 \mu \mathrm{L}$ of Mg ATP were mixed to a solution consisting of $10 \mu \mathrm{L}$ of luciferase, and $10 \mathrm{mM}$ D-luciferin in $75 \mu \mathrm{L}$ of $0.10 \mathrm{M}$ Tris- $\mathrm{HCl}$ buffer $\mathrm{pH}$ 8.0. In the case of the bioluminescence reaction starting with luciferyl-adenylate, $10 \mu \mathrm{L}$ of $\mathrm{LH}_{2} \mathrm{AMP}$ were mixed to a solution consisting of $10 \mu \mathrm{L}$ of luciferase and $85 \mu \mathrm{L}$ of $0.10 \mathrm{M}$ Tris- $\mathrm{HCl}$ buffer $\mathrm{pH}$ 8.5. All measurements were done in triplicate and averages were reported.

\subsection{Luciferyl-adenylate synthesis}

The luciferyl-adenylate was prepared using D-luciferin (SIGMA) and AMP (SIGMA) by following a previously described preparation procedure for D-luciferyl-adenylate [24]. Luciferyl-adenylate was analyzed with silica gel TLC (moving phase: ethyl acetate/ethanol/water (5:3:2), followed by revelation by fluorescence with a UV lamp. Luciferyladenylate displayed yellowish fluorescence with a $R_{f}=0.68$ $\left(\mathrm{R}_{\mathrm{f}(\text { luciferin })}=0.87\right.$ with greenish fluorescence $)$. A luciferyladenylate concentration was estimated from stoichiometric amounts of luciferin and ATP used for its synthesis. According to such estimations, luciferyl-adenylate concentration in stock solution are in the range between 5 to $10 \mathrm{mM}$.

\subsection{Bioluminescence spectra}

Bioluminescence spectra reported here were recorded in Atto LumiSpectra spectroluminometer (Tokyo, Japan) with cooled CCD camera. For the in vitro bioluminescence, $5.0 \mu \mathrm{L}$ of luciferases were mixed with $90 \mu \mathrm{L}$ of $0.10 \mathrm{M}$ Tris- $\mathrm{HCl} \mathrm{pH} 8.0,5 \mu \mathrm{L}$ of specific substrate $(10 \mathrm{mM}$ D-luciferin; luciferyl-adenylate), and $5 \mu \mathrm{L}$ of a solution consisting of $40 \mathrm{mM}$ ATP/80 $\mathrm{mM} \mathrm{MgSO}$. The bioluminescence spectra were measured in triplicate for at least three independent luciferase preparations. The estimated peak error was $\pm 2.5 \mathrm{~nm}$. Above $620 \mathrm{~nm}$ we assumed peak errors of $\pm 3 \mathrm{~nm}$. 


\subsection{Homology modeling}

I-Tasser [25] was used for ab initio modeling with C-terminal sequence deletion of Pyrearinus termitilluminans (Pte-trunc), Phrixotrix vivianii (PvGR-trunc) and Phrixotrix hirtus (PxRE-trunc) luciferases and Zophobas morio likeluciferase (Zop-trunc). These models were evaluated for stability change upon deletion and effects in the presence of 5'-O-[(N-dehydroluciferyl)-sulphamoyl]-adenosine (SLU) at the ligand site. The ligand was docked into protein models using Blind Docking Server-BINDSURF [15], available at: http://bio-hpc.eu/software/blind-docking-server/. The effects of mutations on protein flexibility and conformations were predicted by DynaMut, a web server implemented to analyze and visualize the impact of mutations [26] available at: http://biosig.unimelb.edu.au/dynamut/. Visualization and analyzes of the best model of each luciferase were performed using PyMol's version 1.4.1. [27].

\section{Acknowledgements FAPESP 2010/05426-8, CNPq 401867/2016-1.}

Author contributions VB produced Re and Pte deletion mutants, MC produced and characterized Zop deletion mutant, GFP characterized Pte and RE deletion mutants, AT made and discussed the three-dimensional models, VV idealized the work, prepared the manuscript and produced pxGR deletion mutant.

\section{Compliance with ethical standards}

Conflict of interest The authors declare no conflict of interests.

\section{References}

1. Viviani, V. R. (2002). The origin, diversity, and structure function relationships of insect Luciferases. Cellular and Molecular Life Sciences: CMLS, 59(11), 1833-1850.

2. Day, J. C., Tisi, L. C., \& Bailey, M. J. (2004). Evolution of beetle bioluminescence: the origin of beetle luciferin. Luminescence, 19(1), 8-20.

3. A. M. Gulick, V. J. Starai, A. R. Horswill, K. M. Homick, and J. C. Escalante- Semerena. The 1.75 A Crystal Structure of acetyl-CoA Synthetase Bound to adenosine-5'-propylphosphate and Coenzyme A. Biochemistry, 2003, 42, n. 10, 2866-2873.

4. Wood, K. V. (1995). The chemical mechanism and evolutionary development of beetle bioluminescence. Photochemistry and Photobiology, 62, 662-673.

5. Babbitt, P. C., Kenyon, G. L., Martin, B. M., Charest, H., Slyvestre, M., Scholten, J. D., et al. (1992). Ancestry of the 4-chlorobenzoate dehalogenase: analysis of amino acid sequence identities among families of acyl:adenyl ligases, enoyl-CoA hydratases/ isomerases, and acyl-CoA thioesterases. Biochemistry, 31(24), 5594-5604.

6. Conti, E., Franks, N. P., \& Brick, P. (1996). Crystal structure of firefly luciferase throws light on a superfamily of adenylate-forming enzymes. Structure (London, England), 4(3), 287-298.

7. Nakatsu, T., Ichiyama, S., Hiratake, J., Saldanha, A., Kobashi, N., Sakata, K., \& Kato, H. (2006). Structural basis for the spectral difference in luciferase bioluminescence. Nature, 440(7082), 372-376.

8. Gulick, A. M. (2009). Conformational dynamics in the AcylCoA synthetases, adenylation domains of non-ribosomal peptide synthetases, and firefly luciferase. ACS Chemical Biology, 4(10), 811-827.

9. Branchini, B. R., Southworth, T. L., Murtiashaw, M. H., Magyar, R. A., Gonzalez, S. A., Ruggiero, M. C., \& Stroh, J. G. (2004). An alternative mechanism of bioluminescence color determination in firefly luciferase. Biochemistry, 43(23), 7255-7262.

10. May, J. J., Kessler, N., Marahiel, M. A., \& Stubbs, M. T. (2002). Crystal Structure of DhbE, an archetype for aryl acid activating domains of modular nonribosomal peptide synthetases. Proceedings of the National Academy of Sciences of the United States of America, 99(19), 12120-12125.

11. Branchini, B. R., Southworth, T. L., Murtiashaw, M. H., Wilkinson, S. R., Khattak, N. F., Rosenberg, J. C., \& Zimmer, M. (2005). Mutagenesis evidence that the partial reactions of firefly bioluminescence are catalyzed by different conformations of the luciferase C-terminal domain. Biochemistry, 44(5), 1385-1393.

12. Zako, T., Ayabe, K., Aburatani, T., Kamiya, N., Kitayama, A., Ueda, H., \& Nagamune, T. (2003). Luminescent and substrate binding activities of firefly luciferase $\mathrm{N}$-terminal domain. Biochimica et Biophysica Acta, 1649(2), 183-189.

13. Ayabe, K., Zako, T., \& Ueda, H. (2005). The role of firefly luciferase $\mathrm{N}$-terminal domain in efficient coupling of adenylation and oxidative steps. FEBS Letters, 579, 4389-4394.

14. V.R. Viviani, F.G.C. Arnoldi, B. Venkatesh, A.J.S. Neto, F.G.T. Ogawa, A.T.L. Oehlmeyer, Y. Ohmiya, (2006) Active-site properties of phrixotrix railroad worm green and red bioluminescence-eliciting luciferases. The Journal of Biochemistry 140(4), :467-474

15. I. Sánchez-linares, H. Pérez-Sánchez, J. M. Cecilia, and J. M. García, High-Throughput Parallel Blind Virtual Screening Using BINDSURF. BMC bioinformatics, 2012, 13 Suppl 14, n. Suppl 14, 1471-2105.

16. N. N. Ugarova, and L. Y. Brovko, Protein structure and bioluminescent spectra for firefly bioluminescence. Luminescence: the journal of biological and chemical luminescence, 2002, 17, n. 5, 321-330.

17. Hirano, T., Hasumi, Y., Ohtsuka, K., Maki, S., Niwa, H., Yamaji, M., \& Hashizume, D. (2009). Spectroscopic studies of the lightcolor modulation mechanism of firefly (beetle) bioluminescence. Journal of the American Chemical Society, 131(6), 2385-2396.

18. D. Kato, Firefly Luciferase as Biocatalysts. In: MATSUDA, T. (Ed.). Future Directions in Biocatalysis. 2 ed.: Elsevier, 2017. p. 460.

19. Viviani, V. R., Silva, A. C., Perez, G. L., Santelli, R. V., Bechara, E. J., \& Reinach, F. C. (1999). Cloning and molecular characterization of the cDNA for the Brazilian larval click-beetle Pyrearinus termitilluminans luciferase. Photochemistry and Photobiology, 70(2), 254-260.

20. Viviani, V. R., Prado, R. A., Neves, D. R., Kato, D., \& Barbosa, J. A. R. G. (2013). A route from darkness to light: emergence and evolution of luciferase activity in AMP-CoA-ligases inferred from a mealworm luciferase-like enzyme. Biochemistry, 52(23), 3963-3973.

21. Viviani, V. R., Scorsato, V., Prado, R. A., Pereira, J. G., Niwa, K., Ohmiya, Y., \& Barbosa, J. A. R. G. (2010). The origin of luciferase activity in zophobas mealworm AMP/CoA-ligase (Protoluciferase): luciferin stereoselectivity as a switch for the oxygenase activity. Photochemical Photobiological Sciences, 9(8), 1111-1119.

22. Viviani, V. R., Arnoldi, F. G., Neto, A. J., Oehlmeyer, T. L., Bechara, E. J. H., \& Ohmiya, Y. (2008). The structural origin and 
biological function of $\mathrm{pH}$-sensitivity in firefly luciferases. Photochemical Photobiological Sciences, 7(2), 59-169.

23. V. R. Viviani, A. J. Silva Neto, F. G. Arnoldi, J. A.R.G. Barbosa, and Y. Ohmiya. The influence of the loop between residues 223-235 in beetle luciferase bioluminescence spectra: a solvent gate for the active site of $\mathrm{pH}$-sensitive luciferases. Photochemistry and photobiology, 2008, 84, n. 1, 138-144.

24. Viviani, V. R., \& Ohmiya, Y. (2006). Bovine serum albumin displays luciferase-like activity in presence of luciferyl adenylate: insights on the origin of protoluciferase activity and bioluminescence colours. Luminescence, 21(4), 262-267.
25. Roy, A., Kucukural, A., \& Zhang, Y. (2010). I-TASSER: a unified platform for automated protein structure and function prediction. Nature protocols, 5(4), 725-738.

26. Rodrigues, C. H., Pires, D. E., \& Ascher, D. B. (2018). DynaMut: predicting the impact of mutations on protein conformation, flexibility and stability. Nucleic Acids Research, 46(W1), 350-355.

27. Delano, W. L, The PyMOL Molecular Graphics System. 2008. Disponível em: http://www.pymol.org. 